\title{
Moscow Region Forest Vegetation Database
}

\author{
Elena Tikhonova, Olga Morozova \& Olga Pesterova
}

\begin{abstract}
Relevés of forest vegetation of Querco-Fagetea and Vaccinio-Piceetea classes were monitored between 1996-2011 in the Moscow region. Natural forests as well as forest plantations of different ages were surveyed. Almost all plots were located by GPS. At present about 750 relevés are available in the database. The Moscow Region Forest Vegetation Database (GIVD ID EU-RU-006) is used for biodiversity analyses, vegetation classification and succession studies.
\end{abstract}

Keywords: biodiversity; Querco-Fagetea; Russia; Vaccinio-Piceetea.

\section{Moscow Region Forest Vegetation Database}

Scope: Relevés of forest vegetation of Querco-Fagetea and Vaccinio-Piceetea classes were monitored between 1996-2011 in the Moscow region. Natural forests as well as forest plantations of different ages were surveyed. Almost all plots were located by GPS.

Status: completed and continuing Period: 1987-2011

Database manager(s): Elena Tikhonova (tikhonova.cepl@gmail.com)

Owner: (private)

Web address: [NA]

Availability: according to a specific agreement

Database format(s): TURBOVEG

Online upload: no

Online search: no

Publication: [NA]

Plot type(s): normal plots; time series

Export format(s): TURBOVEG, MS Access, Excel

Plot-size range: $100-400 \mathrm{~m}^{2}$

Non-overlapping plots: 750

Total plot observations: 900

Estimate of existing plots: [NA]

Number of sources: [NA]

Completeness: [NA]

Valid taxa: [NA]

Countries: RU: $100.0 \%$

Forest: $93 \%$ - Non-forest: aquatic: $0 \%$; semi-aquatic: $0 \%$; arctic-alpine: $0 \%$; natural: $0 \%$; semi-natural: $7 \%$; anthropogenic: $0 \%$

Guilds: all vascular plants: $100 \%$; bryophytes (terricolous or aquatic): $80 \%$

Environmental data: altitude: $60 \%$; slope aspect: $80 \%$; slope inclination: $80 \%$; microrelief: $85 \%$; soil depth: $50 \%$; surface cover other than plants (open soil, litter, bare rock etc.): $60 \%$; other soil attributes: $60 \%$

Performance measure(s): cover: $100 \%$; measurements like diameter or height of trees: $70 \%$

Geographic localisation: GPS coordinates (precision $25 \mathrm{~m}$ or less): $95 \%$; point coordinates less precise than GPS, up to $1 \mathrm{~km}: 5 \%$

Sampling periods: $1980-1989: 15.0 \%$; 1990-1999: 15.0\%; 2000-2009: 50.0\%; 2010-2019: $20.0 \%$

Information as of 2012-07-12; further details and future updates available from http://www.givd.info/ID/EU-RU-006

Elena Tikhonova* (tikhonova.cepl@gmail.com), Olga Morozova (moroz_ov@orc.ru), Olga Pesterova (Olga1hbg@yandex.ru) Center for Forest Ecology and Productivity RAS, Moscow, RUSSIA

*Corresponding author 